Supporting Information for

\title{
Polarity tunable trionic electroluminescence in monolayer $\mathrm{WSe}_{2}$
}

Junyong Wang ${ }^{1,2}$, Fanrong Lin ${ }^{1,2}$, Ivan Verzhbitskiy ${ }^{1,2}$, Kenji Watanabe ${ }^{3}$, Takashi Taniguchi $^{3}$, Jens Martin ${ }^{1,2}$, Goki Eda ${ }^{1,2,4, *}$

${ }^{1}$ Department of Physics, National University of Singapore, 2 Science Drive 3, Singapore 117542

${ }^{2}$ Centre for Advanced 2D Materials, National University of Singapore, 6 Science Drive 2, Singapore 117546

${ }^{3}$ National Institute for Material Science, 1-1 Namiki, Tsukuba 305-0044, Japan

${ }^{4}$ Department of Chemistry, National University of Singapore, 3 Science Drive 3, Singapore 117543

* Corresponding author: g.eda@ nus.edu.sg 


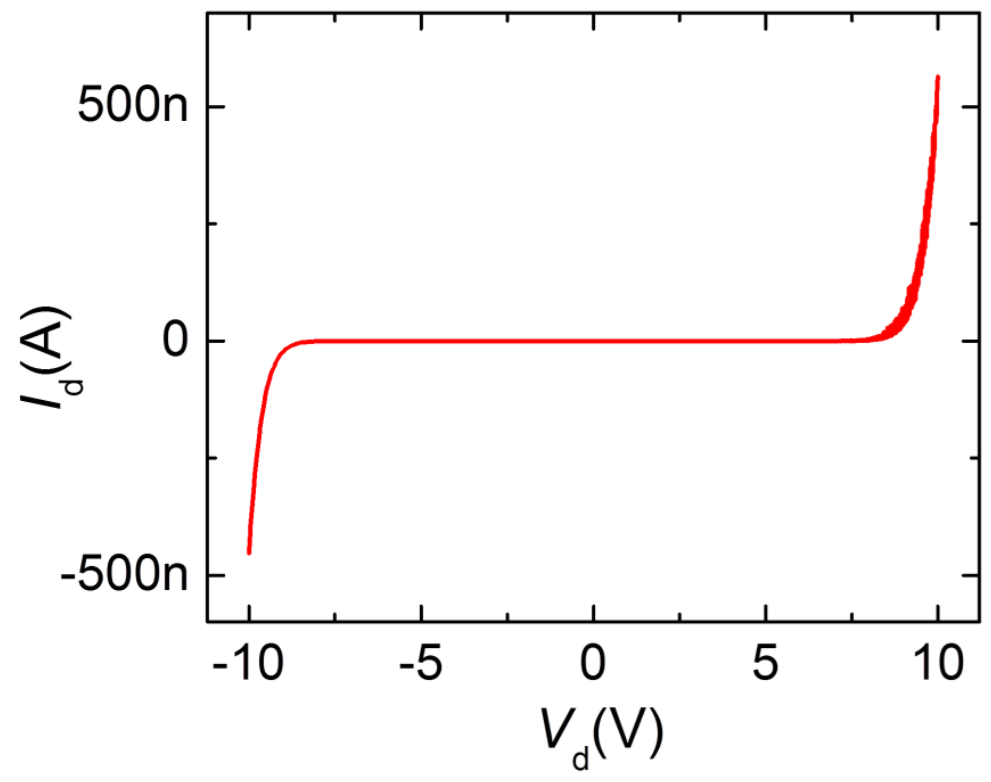

Figure S1. Tunneling characteristic of a metal-insulator-metal (MIM) device consisting of graphene/hBN/graphene. It shows the symmetric tunneling behavior in both polarities without rectifying behavior, which is in contrast with metal-insulator-semiconductor (MIS) devices. 
a
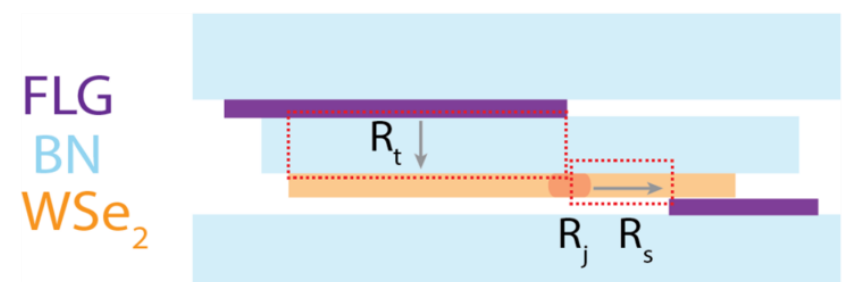

b
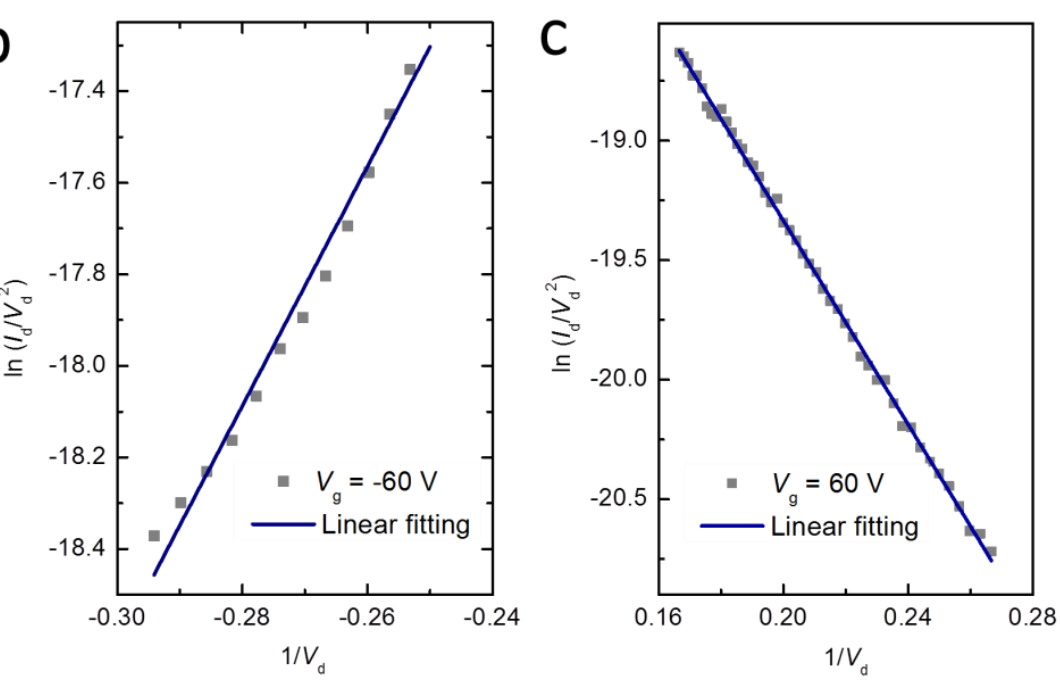

Figure S2. (a) A schematic cartoon showing the total resistance contributed from tunneling resistance $R_{\mathrm{t}}$, sheet resistance $R_{\mathrm{s}}$ and junction resistance $R_{\mathrm{j}}$ in the MIS device. (b-c) FowlerNordheim plot of the $I_{\mathrm{d}}-V_{\mathrm{d}}$ characteristic in the tunneling resistance dominated regimes at $V_{\mathrm{g}}$ $=-60 \mathrm{~V}$ and $V_{\mathrm{g}}=60 \mathrm{~V}$. 


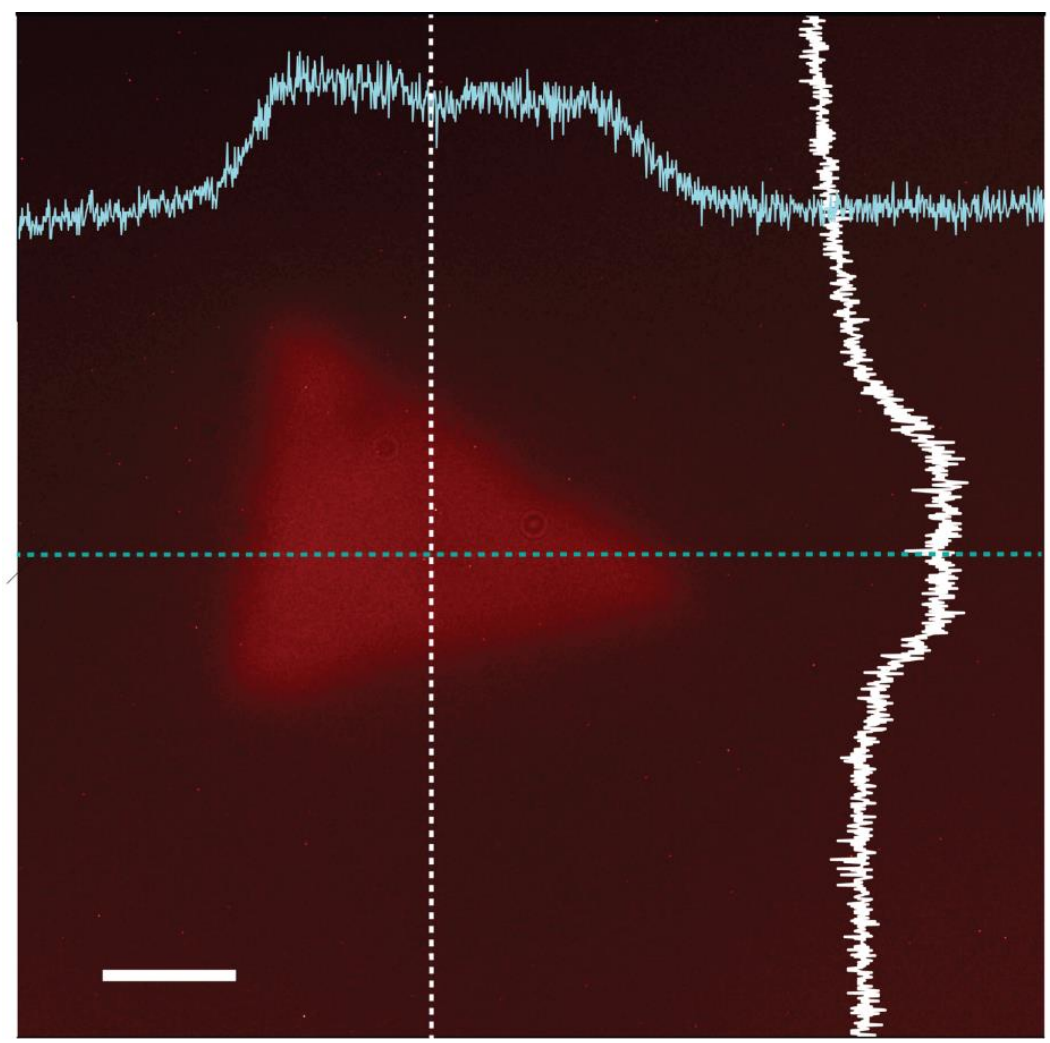

Figure S3. Profile of the intensities of the electroluminescence (EL) from the device. This image clearly shows the uniform emission from the whole MIS device and rules out the emission from edges or contacts area. The scale bar is $5 \mu \mathrm{m}$. 
a

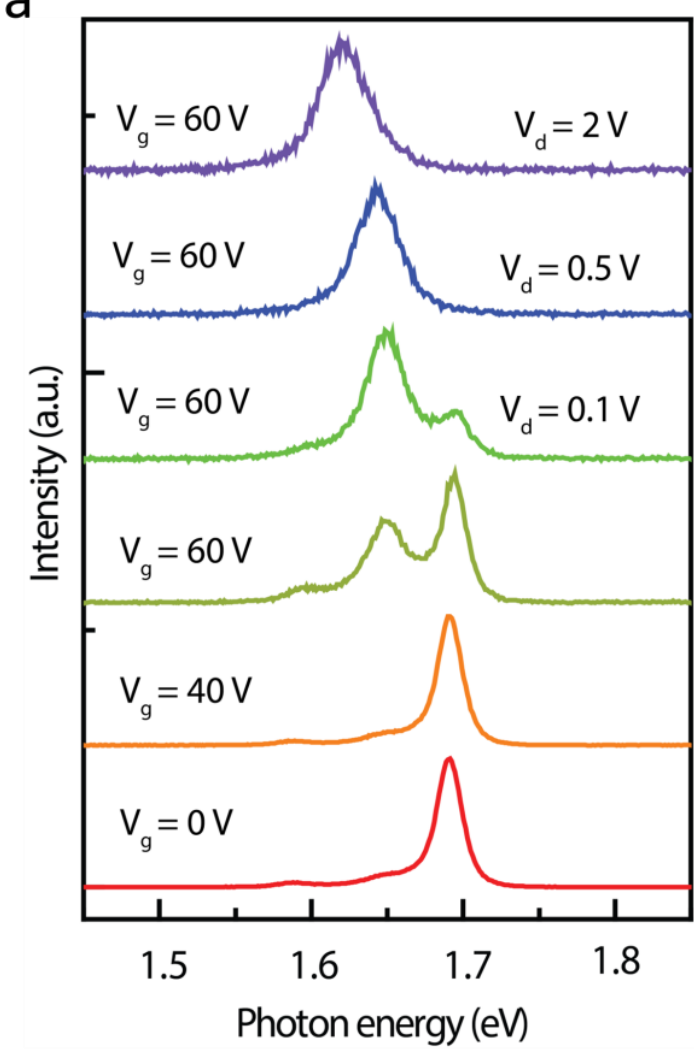

b

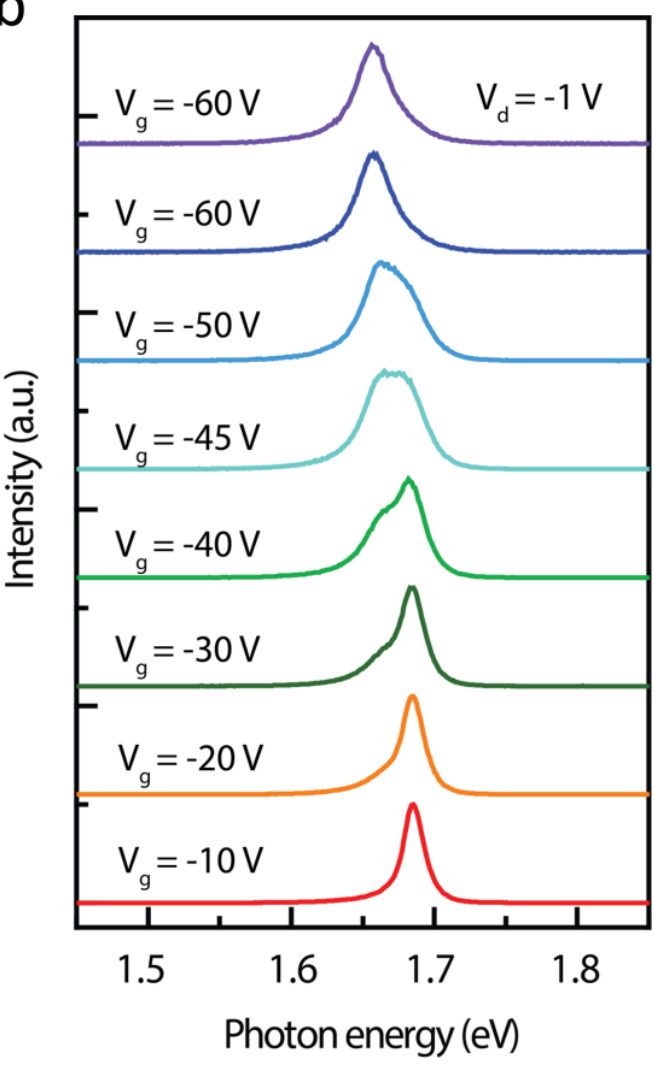

Figure S4. PL spectra of the monolayer $\mathrm{WSe}_{2}$ as a function of backgate $\left(V_{\mathrm{g}}\right)$ and bias $\left(V_{\mathrm{d}}\right)$ voltages at $80 \mathrm{~K}$. The emission from neutral exciton reduces with increased voltages and trions dominate the PL at heavily doped regimes. 

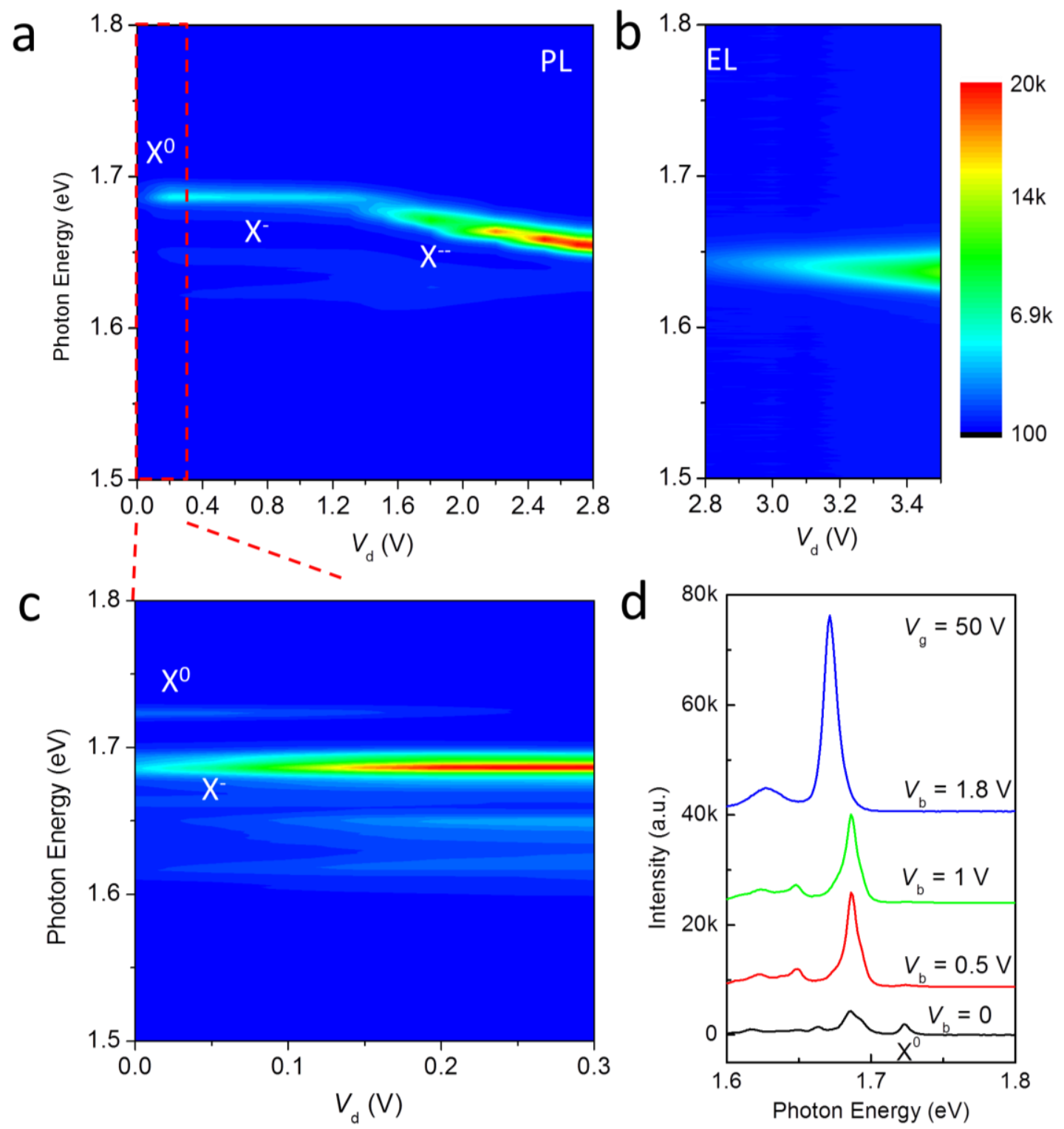

Figure S5. (a) PL of monolayer $\mathrm{WSe}_{2}$ in the positive bias $\left(V_{\mathrm{d}}\right)$ regime at $10 \mathrm{~K}$. Fine structure of the negative trion $\left(\mathrm{X}^{-}, \mathrm{X}^{--}\right)$can be resolved. The weak neutral exciton $\left(\mathrm{X}^{0}\right)$ emission compared with the trion emission is shown in (c). (b) EL from negative trions as a function of bias voltage $V_{\mathrm{d}}$ above the threshold voltage. (c) Zoom in of the bias dependent PL in (a) from 0 to $0.3 \mathrm{~V}$. (d) PL spectra evolution of monolayer $\mathrm{WSe}_{2}$ with the positive bias. 

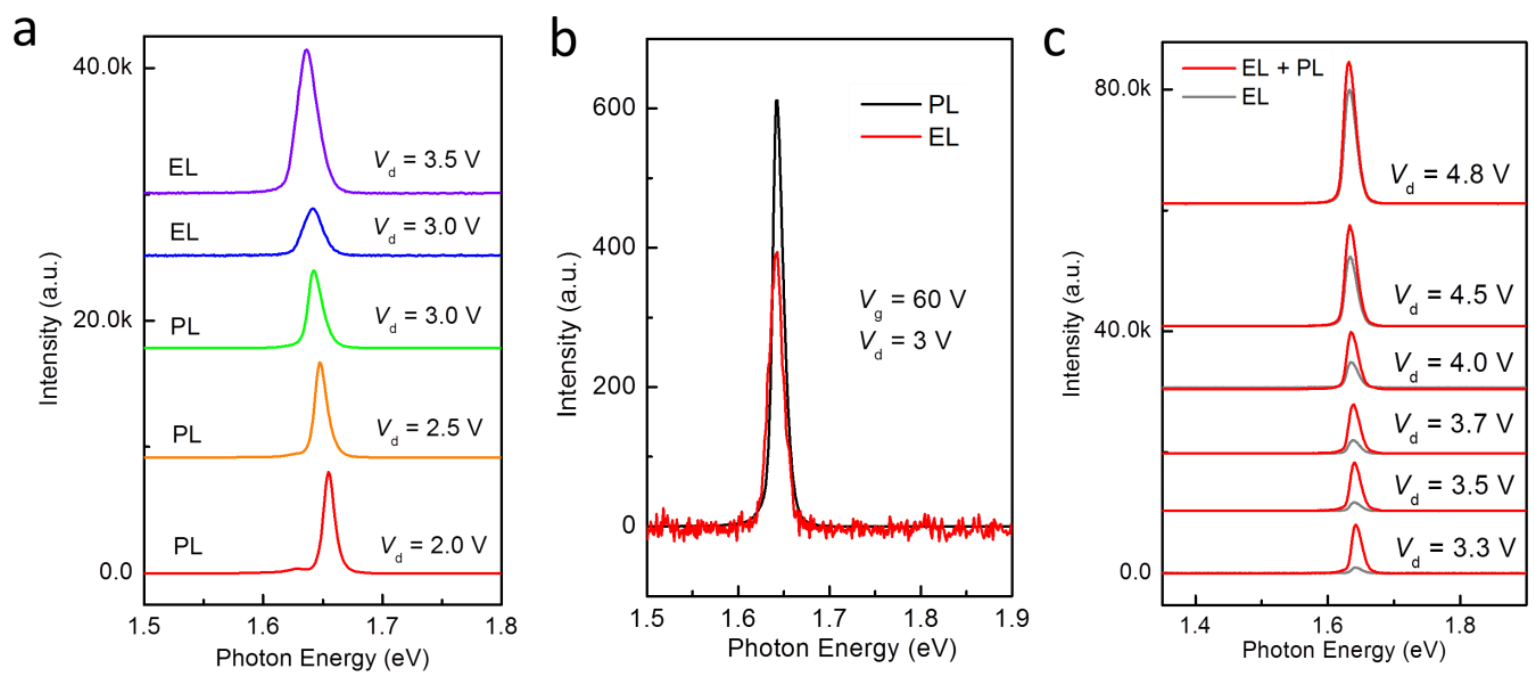

Figure S6. (a) PL and EL spectra near the threshold voltage at positive gate and bias at $10 \mathrm{~K}$. (b) PL and EL near the threshold bias highlighting similarity of the emission spectra. (c) Emission from the device with/without laser light excitation at various bias conditions. 

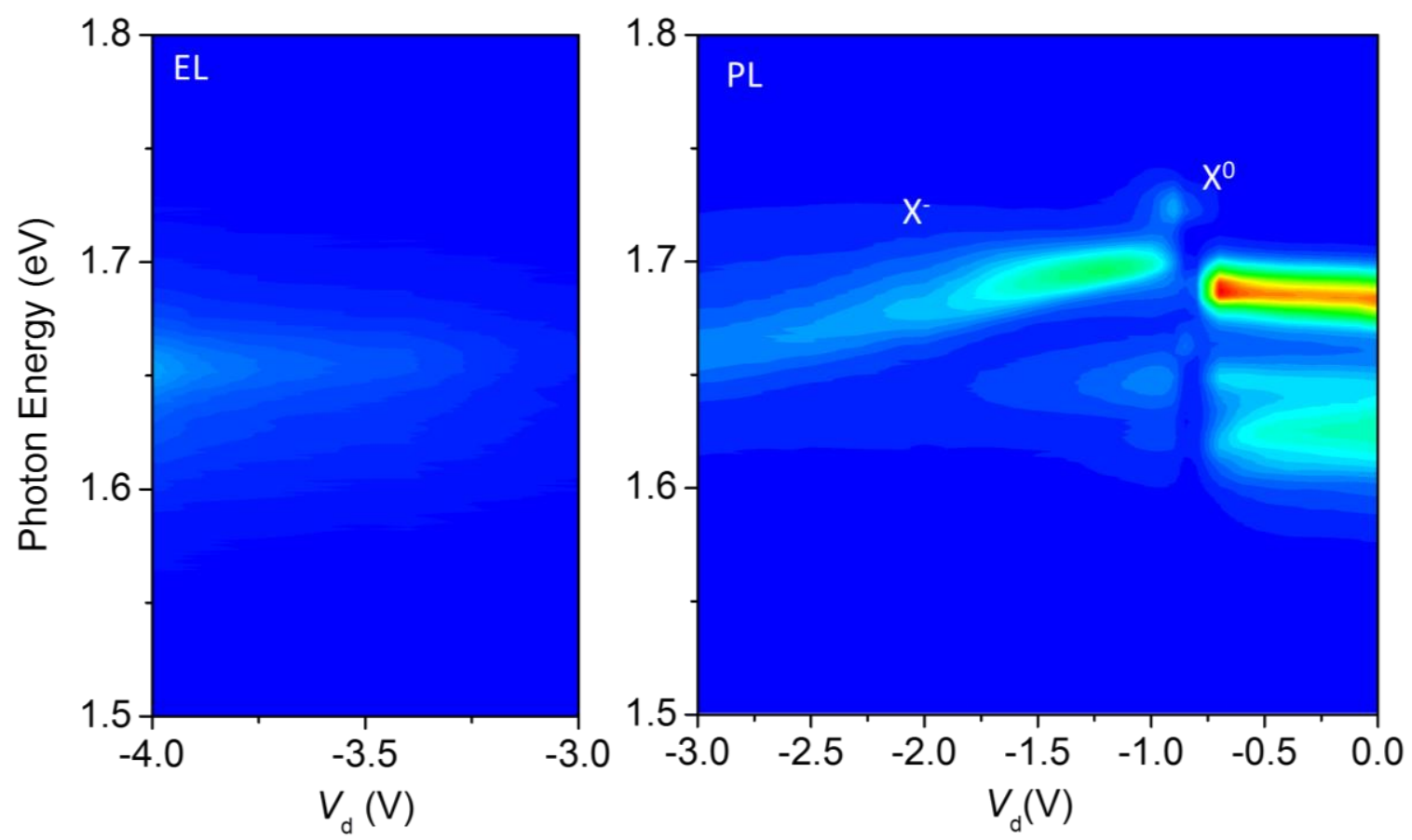

Figure S7. Bias dependent EL (left) and PL (bias) of the monolayer $\mathrm{WSe}_{2}$ in the negative bias condition at $10 \mathrm{~K}$. 

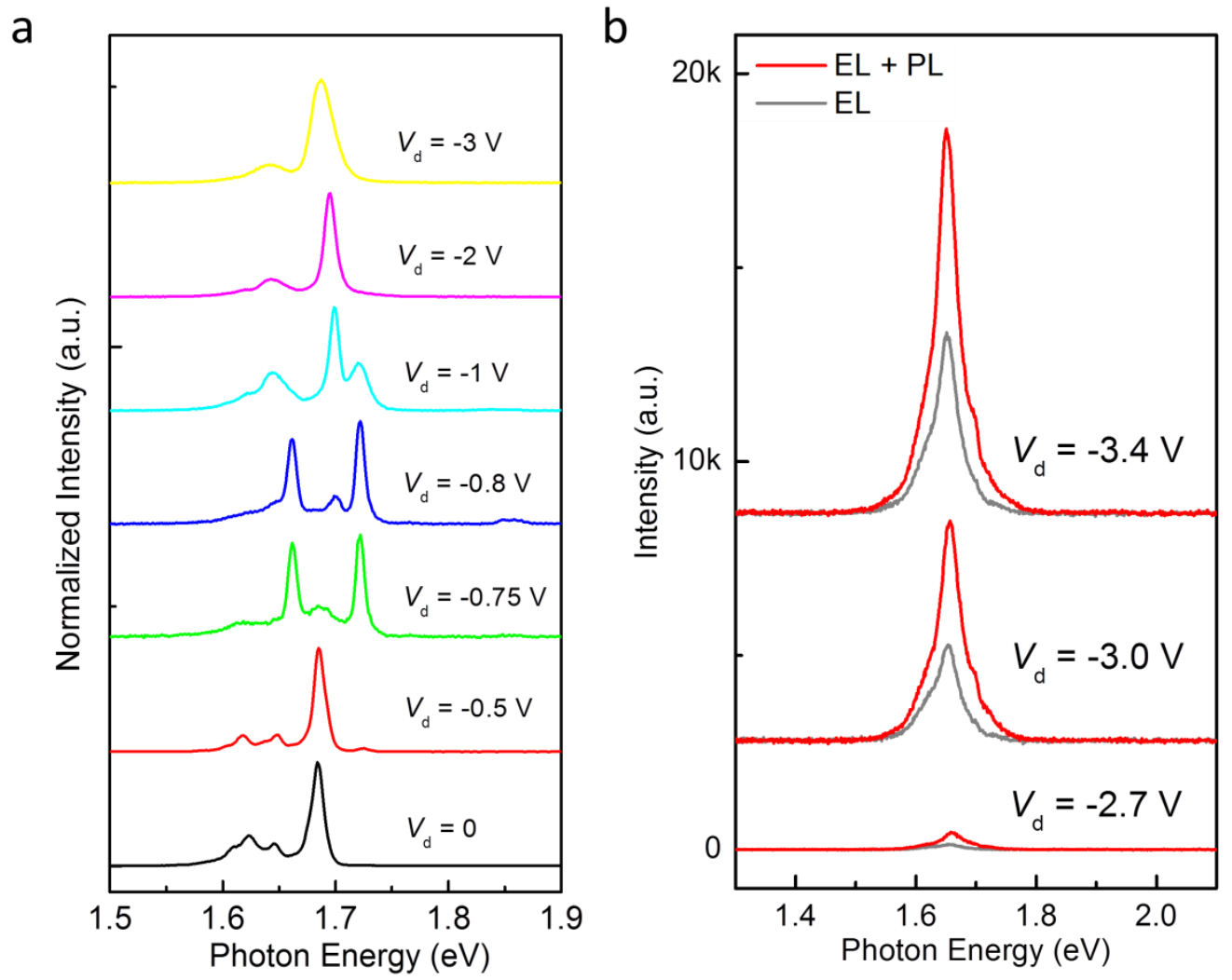

Figure S8. (a) Spectral evolution of the PL at the negative bias. At $V_{\mathrm{d}}=0$, the emission is comprised of the low-energy defect-activated peaks and negative trion. The latter could be explained by the trapped carriers left in the $\mathrm{WSe}_{2}$ after positive bias sweep performed earlier on the device. With increasing negative voltage biases, negative trion peak intensity reduces, while neutral peak emerges at $V_{\mathrm{d}}=-0.75 \mathrm{~V}$. Further increase in negative voltage quench the negative trion with subsequent rise of positive trion at $V_{\mathrm{d}}=-1 \mathrm{~V}$. (b) Emission from the device with/without light excitation at various bias conditions. 

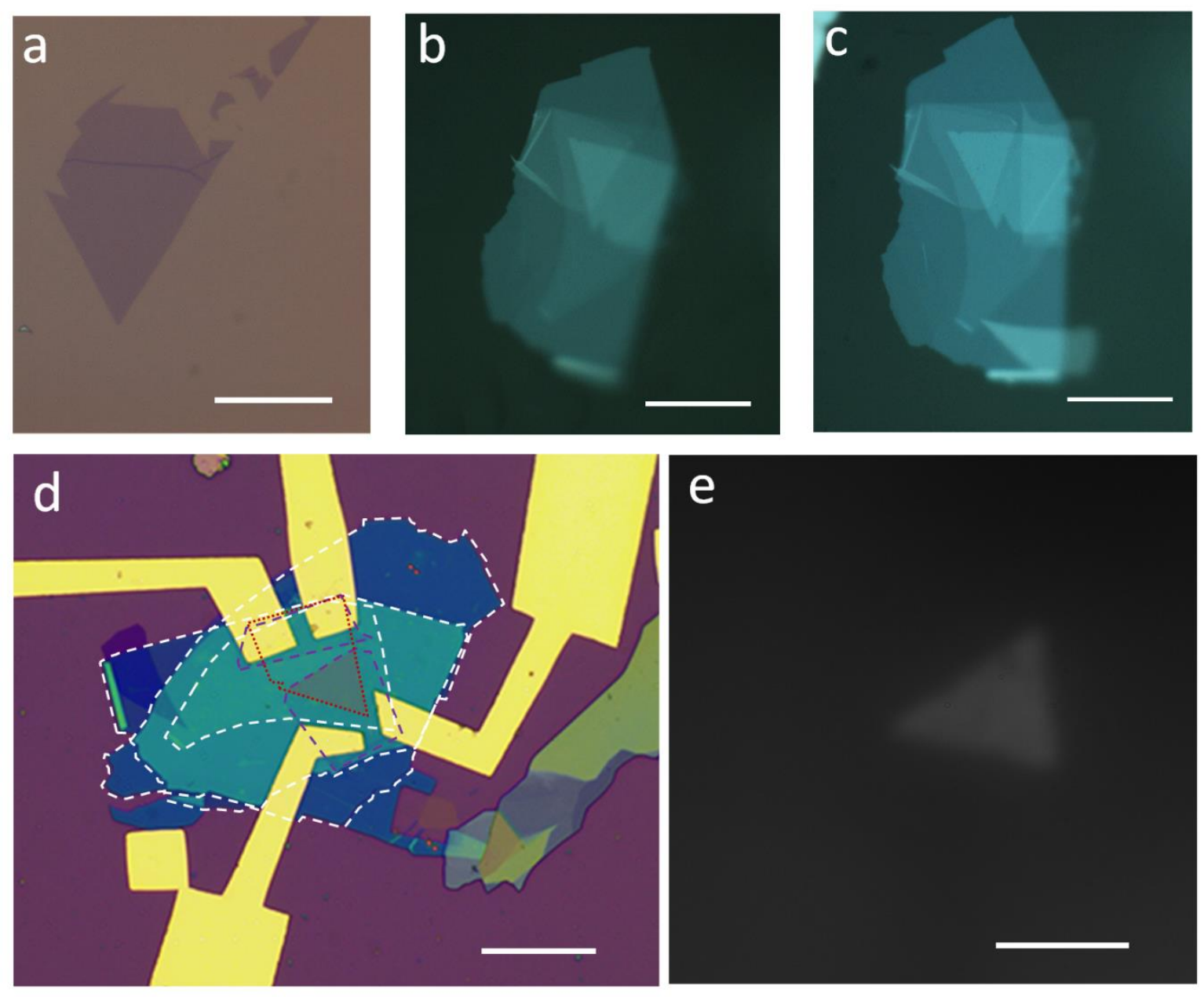

Figure S9. Device fabrication processes. (a) Optical image of the monolayer $\mathrm{WSe}_{2}$ exfoliated on $\mathrm{Si} / \mathrm{SiO}_{2}$ substrate. (b) Optical image of the $1 \mathrm{~L}-\mathrm{WSe} \mathrm{e}_{2} /$ thin-hBN/FLG/top-hBN flake on the PPC/PDMS stamp. (c) Optical image of the device in (b) with additional FLG layer as a contact to $1 \mathrm{~L}-\mathrm{WSe}_{2}$. (d) True color optical image of the ready device with outlines of the $\mathrm{vdW}$ heterostack. White, purple and red lines are hBN, FLGs and monolayer $\mathrm{WSe}_{2}$, respectively. Shadowed area is the active MIS device. (e) Optical image of the EL emission from the active area. All scale bars are $20 \mu \mathrm{m}$. 


\section{Total resistance and rectifying characterise in the MIS device}

In our MIS devices the spatial overlap of FLGs in the heterostack (tunneling FLG and contact FLG) is avoided to circumvent carrier tunneling between these layers. Thus, a small portion of the monolayer $\mathrm{WSe}_{2}$ between the junction area and FLG contact contributes to the lateral sheet resistance $R_{\mathrm{s}}$, which can be tuned by the back gate only. This resistance is in series with the tunneling resistance $R_{\mathrm{t}}$ of the overlap region as shown in Figure $\mathrm{S} 2$. When a bias is applied to FLG, the carrier density in this region is different from the region that is electrostatically doped by both the backgate and the bias. Thus, when the bias is applied between the source and drain terminals, the resistance mainly consists of three contributions: 1) the lateral sheet resistance of $\left.\mathrm{WSe}_{2}, R_{\mathrm{s}} ; 2\right)$ the junction resistance $R_{\mathrm{j}}$ from the junction between monolayer $\mathrm{WSe}_{2}$ with different doping levels; and 3) the tunneling resistance $R_{\mathrm{t}}$ of the MIS junction. The contact resistance at the $\mathrm{Au} / \mathrm{Cr} / \mathrm{FLG}$ junction and the sheet resistance of the FLG are orders of magnitude less than the above mentioned contributions. Thus we will not consider it in the following discussions.

At $60 \mathrm{~V}$ backgate, monolayer $\mathrm{WSe}_{2}$ is in the $n$-type conducting regime, as evident from the transfer curve. When a positive bias $V_{\mathrm{d}}$ is applied, $\mathrm{WSe}_{2}$ below FLG/hBN is further $n$-doped, and when the bias exceeds a threshold, tunnel current emerges. The threshold voltage, which is largely determined by the voltage drop across both, the tunneling layer and the uncovered $\mathrm{WSe}_{2}$ sheet, depends on the thickness of the thin $\mathrm{hBN}$ layer and conductance of monolayer $\mathrm{WSe}_{2}$. At a large positive backgate and positive bias, $\mathrm{WSe}_{2}$ is heavily $n$-doped, and $R_{\mathrm{S}}$ is small compared to the tunneling resistance. Meanwhile, a junction resistance $R_{\mathrm{j}}$ from the $n-n^{+}$junction is also expected to be small. ${ }^{1}$ Hence, in this condition, the total resistance is dominated by the tunneling resistance through $\mathrm{hBN}$ and EL is observed in this regime. 
In the negative diode bias condition, the $n$-doping of $\mathrm{WSe}_{2}$ due to positive backgate is compensated by the bias. In this condition, the lateral junction resistance between the singleand double-gated regions can be significant. At a high negative bias, $\mathrm{WSe}_{2}$ will be $p$-doped despite the positive backgate. Thus, a lateral $p-n$ junction is formed. This $p$ - $n$ junction is in reverse bias when $V_{\mathrm{d}}<0$, rendering the high resistance of the device. This contributes to the rectifying characteristic at negative biases.

The rectifying behaviours in the negative backgate condition can be explained in a similar way due of the ambipolar properties of $\mathrm{WSe}_{2}$.

\section{F-N tunneling analysis}

In this Fowler-Nordheim tunneling regime, field emission current dominate over direct tunneling. Based on the Fowler-Nordheim (F-N) tunneling model,

$$
I\left(V_{d}\right)=\frac{A_{e f f} q^{3} m V_{d}^{2}}{8 \pi h \phi_{B} d^{2} m^{*}} \exp \left(\frac{-8 \pi \sqrt{2 m^{*}} \phi_{B}^{3 / 2} d}{3 h q V_{d}}\right)
$$

where $A_{e f f}, \phi_{B}, q, m, m^{*}, d$ and $h$ are effective contact area, barrier height, electron charge, free electron mass, effective electron mass, barrier width (hBN thickness) and Plank's constant, respectively. Where $m * / m=0.26$ for electrons and 0.47 for holes in $\mathrm{hBN}^{2,3}$

The slope in the linear regime of the $\ln \left(I / V_{d}^{2}\right)$ versus $1 / V_{d}$ plot (F-N plot) is

$$
k=\frac{-8 \pi \sqrt{2 m^{*}} \phi_{B}^{3 / 2} d}{3 h q}
$$

A linear dependence in Fowler-Nordheim $(\mathrm{F}-\mathrm{N})$ plot will indicate the field emission tunneling through hBN. At high gate and bias condition where the tunneling resistance dominates, the tunneling behavior can be well fitted by the F-N tunneling model, as shown in Figure S2 (b-c).

Based on the electron affinity and the bandgap of each layer in the MIS structures, we constructed the band diagram for FLG/hBN/WSe 2 at different bias conditions (Figures $1 \mathrm{c}$ and 
4, main text). In this device ( $\mathrm{hBN}$ is $\sim 3 \mathrm{~nm}$ ), the obtained barrier heights are $1.32 \mathrm{eV}$ for positive biases (holes) and $-1.89 \mathrm{eV}$ for negative biases (electrons) based on the fitting of the slope for positive and negative charge tunneling. Here, we assume that the carriers from FLG are responsible for the field emission. The extracted barriers are smaller than the tunneling barrier heights estimated from the band diagram. The discrepancy may be due to additional resistance components of the semiconductor and possible carrier flow from $\mathrm{WSe}_{2}$ to FLG. ${ }^{4}$

\section{Calculation of doping level and Fermi level shift}

In a field effect transistor configuration, the charge-carrier density is controlled by the capacitive coupling to a gate,

$$
n=C\left(V_{g}-V_{t h}\right) / e
$$

where $n$ is the carrier concentration and $\mathrm{C}$ is the geometrical capacitance.

For single-layer $\mathrm{WSe}_{2}$, in back gate, there are two capacitors in series. One is the backgate from $\mathrm{SiO}_{2}$, the other one is from the bottom $\mathrm{BN}$.

$$
C_{B G-S i O_{2}}=\varepsilon \varepsilon_{0} / d_{B G-S i O_{2}}
$$

where $\varepsilon$ is the dielectric constant of $\mathrm{SiO}_{2}(\sim 4), \varepsilon_{0}$ is the permittivity of free space and $d_{\mathrm{BG}}$ $\mathrm{SiO} 2$ is $285 \mathrm{~nm}$.

$$
C_{B G-B N}=\varepsilon \varepsilon_{0} / d_{B G-B N}
$$

where $\varepsilon$ is the dielectric constant of $\mathrm{BN}(\sim 3.3-3.8), \varepsilon_{0}$ is the permittivity of free space and $d_{\mathrm{BG}-\mathrm{BN}}$ is $25 \mathrm{~nm} .^{5}$

The capacitance is the series combination of these two

$$
C_{B G}{ }^{-1}=C_{B G-B N}{ }^{-1}+C_{B G-S i O_{2}}{ }^{-1}
$$

With the effective of top bias, 
$C_{T G}=\varepsilon \varepsilon_{0} / d_{B N-\text { tunnel }}$

Based on this estimation, we obtained that the majorities in $\mathrm{WSe}_{2}$ at bottom gate $60 \mathrm{~V}$ and top gate $4 \mathrm{~V}$ is $2.5 \times 10^{13} \mathrm{~cm}^{-2}$, which is a much higher density than the non-equilibrium minors through tunneling injection.

Next we estimate the Fermi level shifts in FLG and $\mathrm{WSe}_{2}$ in the bias condition. For electrons in 2D, the density of state (DOS) is given by

$$
\operatorname{DOS}(2 D)=\frac{m}{\pi \hbar^{2}}
$$

which is approximately $0.4 \times 10^{15} /\left(\mathrm{cm}^{2} \bullet \mathrm{eV}\right)$. The electron mass would need to be replaced by the effective mass. The effective mass of $\mathrm{WSe}_{2}$ is about $0.35 .^{6} \mathrm{We}$ model a bilayer graphene with an effective mass of about $0.04 .^{7}$ The DOS of bilayer graphene is expected to be at least a factor 8 smaller than DOS of $\mathrm{WSe}_{2}$. For an induced density of $10^{13} \mathrm{~cm}^{-2}$, the Fermi-level of $\mathrm{WSe}_{2}$ would be shifted by about $100 \mathrm{meV}$, while for bilayer graphene the shift would be around $800 \mathrm{meV}$. Indeed, there is some uncertainty in the DOS, and also in the reported $m^{*}$. However, the effect of difference in Fermi level shift is robust. Since the Fermilevel in FLG shifts more than in $\mathrm{WSe}_{2}$, the tunneling barrier for charge carriers originating from FLG is reduced accordingly. Since FN-tunneling depends very sensitive to barrier height, we observed the nearly unipolar tunneling.

\section{PL and EL analysis at $10 \mathrm{~K}$}

We have performed measurements of PL and EL at $10 \mathrm{~K}$, where excitonic features of $\mathrm{WSe}_{2}$ generally show substantially narrower peaks due to reduced inhomogeneous broadening. Figure S5a shows 2D contour map of the PL at positive voltage biases, where emission from two well-resolved negative trion peaks $\left(\mathrm{X}^{-}\right.$and $\left.\mathrm{X}^{--}\right)$can be clearly seen. This splitting is attributed to the difference in binding energies of inter- (high energetic branch) 
and intra-valley (low energetic branch) trion configurations. ${ }^{8,9}$ The EL (Figure S5b) emission emerges at a high biases, where the intra-valley trion $\left(\mathrm{X}^{--}\right)$already dominates the spectrum.

Similarly, with negative backgate and biases, the EL and PL emission spectra are dominated by positive trions, as shown in Figure S7. At low temperatures, emission from bound excitons (defect-activated) is known to play a significant role in the PL spectrum of $\mathrm{WSe}_{2}$. Bound excitons can be distinguished from free excitonic emissions (neutral exciton, trion, biexciton and charged biexciton) from the photon energy, gate- and power-dependence. It is worth noting that in high doping regime the intensities of bound exciton peaks are substantially reduced, which allowed us to obtain emission purely from trions (Figures S5 and S7).

Since the trion emission peak continuously shifts with doping level, it is crucial to compare EL and PL emission spectra at the same bias conditions. Figures S6c and S8b show the measured emission with and without photoexcitation at the same doping levels. We found the emission peak energy and line-shape of the electrically generated (EL) and photogenerated (PL) trions to be identical, thus validating our assignments.

\section{Quantum efficiency calculation}

EL external quantum efficiency (EQE, the number of photons emitted per each electron-hole pair electrically generated) in our MIS devices was estimated with the reference fluorescent dye. ${ }^{10}$ The dye exhibited PL quantum yield (QY, the number of photons emitted per number of photons absorbed) of $\sim 36 \%$. PL intensities of the reference sample and $\mathrm{WSe}_{2}$ were measured in our laser confocal microscope, while keeping all experimental conditions strictly identical. For the determination of EL EQE, we need to consider the emission area and the collection efficiency, which are different from the case of PL. Indeed, restrictions 
from the objective lens and confocal optical setup would reduce the collection efficiency from the extended emission areas. Emitters located away from the centre of the field of view would have lower collection efficiency as compared to those located in the centre. While PL emission area usually do not largely exceed the size of the laser spot $\left(\sim 1 \mu \mathrm{m}^{2}\right)$, EL emission in our devices nearly uniformly span over areas $>200 \mu \mathrm{m}^{2}$. Taking into account the difference in emission areas but assuming the equal collection efficiency for PL and EL, our estimation yields the lower bound for the EL quantum efficiency. 


\section{Supporting References}

1. Baugher, B. W.; Churchill, H. O.; Yang, Y.; Jarillo-Herrero, P. Nat. Nanotechnol. 2014, 9, (4), 262.

2. $\quad$ Lee, G.-H.; Yu, Y.-J.; Lee, C.; Dean, C.; Shepard, K. L.; Kim, P.; Hone, J. Appl. Phys. Lett. 2011, 99, (24), 243114.

3. Britnell, L.; Gorbachev, R. V.; Jalil, R.; Belle, B. D.; Schedin, F.; Katsnelson, M. I.; Eaves, L.; Morozov, S. V.; Mayorov, A. S.; Peres, N. M. Nano Lett. 2012, 12, (3), 1707-1710. 4. Vu, Q. A.; Lee, J. H.; Nguyen, V. L.; Shin, Y. S.; Lim, S. C.; Lee, K.; Heo, J.; Park, S.; Kim, K.; Lee, Y. H.; Yu, W. J. Nano Lett. 2017, 17, (1), 453-459.

5. Laturia, A.; Van de Put, M. L.; Vandenberghe, W. G. NPJ 2D Mater. Appl. 2018, 2, $(1), 6$.

6. Shi, H.; Pan, H.; Zhang, Y.-W.; Yakobson, B. I. Phys. Rev. B 2013, 87, (15), 155304.

7. $\quad$ Zou, K.; Hong, X.; Zhu, J. Phys. Rev. B 2011, 84, (8), 085408.

8. Jones, A. M.; Yu, H.; Ghimire, N. J.; Wu, S.; Aivazian, G.; Ross, J. S.; Zhao, B.; Yan, J.; Mandrus, D. G.; Xiao, D.; Yao, W.; Xu, X. Nat. Nanotechnol. 2013, 8, (9), 634-8.

9. Plechinger, G.; Nagler, P.; Arora, A.; Schmidt, R.; Chernikov, A.; Del Águila, A. G.; Christianen, P. C.; Bratschitsch, R.; Schüller, C.; Korn, T. Nat. Commun. 2016, 7, 12715.

10. Mohamed, N. B.; Wang, F.; Lim, H. E.; Zhang, W.; Koirala, S.; Mouri, S.; Miyauchi, Y.; Matsuda, K. Phys. Status Solidi B 2017, 254, (2), 1600563. 